\title{
Editorial: \\ The Continuing Surprise: Recent Scientific Advances
}

\author{
C. C. Chancey, Editor \\ American Journal of Undergraduate Research \\ University of Northern lowa \\ Cedar Falls, lowa 50614-0150 USA
}

The past week has seen two announcements of scientific advances that have engendered a good deal of surprise, especially for people outside science.

First, scientists of the DZero collaboration at the US Department of Energy's Fermi National Accelerator Laboratory announced evidence for a significant violation of matter-antimatter symmetry in the behavior of fundamental particles. These particles are the building blocks of our physical universe, and any fundamental asymmetry sets basic constraints for how our universe evolved. Physicists have wondered for decades how our "matter" universe came to be: The Standard Model of particle physics-the reigning theory of matter-predicts a very close balance in matter and antimatter. But any balance between matter and antimatter should have caused the early universe to annihilate itself as the matter and antimatter combined. The problem is obvious: we, our world, the universe are still here, and we're all composed of matter.

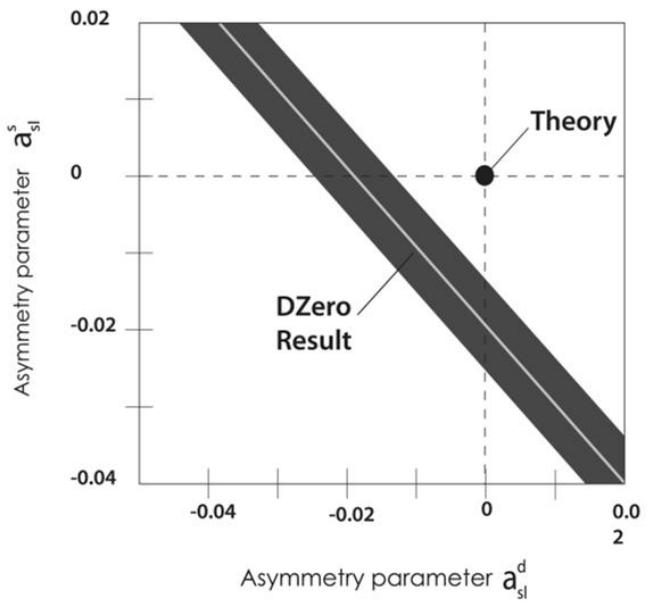

The dominance of matter that we observe in the universe is possible only if there are differences in the behavior of particles and antiparticles. Although physicists have observed such differences (called "CP violation") in particle behavior for decades, these known differences are much too small to explain the observed dominance of matter over antimatter in the universe and are fully consistent with the Standard Model. If confirmed by further observations and analysis, the effect seen by DZero physicists could represent another step towards understanding the observed matter dominance by pointing to new physics phenomena beyond what we know today.

Second, Dr. Craig Venter-the genome pioneer-announced another step in his quest to create synthetic life, by synthesizing an entire bacterial genome and using it to take over a cell. If this sounds a bit Frankensteinish it's not, though the biotechnology achievement is impressive. Venter and his colleagues created a specialized version of the bacterium's genome, and inserted it into a bacterium which had been disabled by having its DNA instructions removed. Venter's actioninserting the specialized genome-started cell division, and it is in this sense that he and his colleagues have "created synthetic life."

We might be concerned at how this technology will develop-as scientists, we should be thoughtful about social implications-but the vista that opens up with this first step in synthetic life is breathtaking. An exciting two weeks! 